\title{
Estimating Surplus Production and Maximum Sustainable Yield from Biomass Data when Catch and Effort Time Series are not Available
}

\author{
S. GARCIA, P. SPARRE and J. CSIRKE
}

Fisheries Department, Food and Agriculture Organization of the United Nations, via delle Terme Caracalla, 00100 Rome (Italy)

(Accepted for publication 30 January 1989)

\begin{abstract}
Garcia, S., Sparre, P. and Csirke, J., 1989. Estimating surplus production and maximum sustainable yield from biomass data when catch and effort time series are not available. Fish. Res., 8: 13-23.

In order to describe a simple method of estimating maximum sustainable yield (MSY), we first demonstrate that the parameters of the two well-known surplus production models of Schaefer and Fox can be expressed in terms of fishing mortality that yields maximum sustainable yield $\left(F_{\mathrm{MSY}}\right)$, annual yield and mean biomass. If $F_{\mathrm{MSY}}$ is known, or, alternatively, is assumed to be some specified function of a known natural mortality rate, it thereby becomes possible to estimate MSY and describe the surplus production function even when the data are limited to one year of estimates for catch and mean biomass. The method is compared to other simple methods for estimating MSY and its potential application is discussed.
\end{abstract}

\section{INTRODUCTION}

In spite of the advances made in the field of fishery sciences and the establishment by most fishery research institutions of data bases for stock assessment purposes, there are still situations where no catch and effort time series exist. In such cases estimation of potential yield is usually based on rough estimates of standing stocks (e.g. from resource surveys) and the general knowledge of some biological characteristics of the species.

Two equations have been proposed in the past to estimate the potential yield of unexploited (Gulland, 1971, p. 2) and exploited (Cadima, in Troadec, 1977) fish stocks for which only estimates of overall biomass and natural mortality are available. Although these were proposed for data-limited situations where the results may be neither very accurate nor precise, the simplicity and ease of application contributed to their extended use. In this paper we discuss the 
requirements and sources of bias of these two equations and show the limitations of Cadima's estimator for exploited stocks. Furthermore,we propose an alternative set of simple formulae with which to estimate maximum sustainable yield (MSY) of exploited stocks and draw the surplus production curve using estimates of total biomass and catch during one year as input. The alternatives are based on the most widely used surplus production models of Schaefer (1954) and Fox (1970).

\section{GULLAND'S FORMULA}

Gulland (1971) proposed the following estimator of the MSY of a virgin stock when estimates of the natural mortality rate $(M)$ and the biomass of the virgin stock $\left(B_{\circ}\right)$ are available:

$\mathrm{MSY}=0.5 M B_{\text {。 }}$

This estimator has been extensively used with variable success. It is based on the observation that in the Schaefer (1954) production model, the biomass at MSY is equal to half the biomass in the virgin state $\left(B_{\mathrm{MSY}}=0.5 B_{\mathrm{o}}\right)$. It is also based on the assumption already made by Tiurin (1962) and Alverson and Pereyra (1969) that the fishing mortality $\left(F_{\mathrm{MSY}}\right)$ at MSY is roughly equal to $M$. It follows that $\mathrm{MSY}=F_{\mathrm{MSY}} B_{\mathrm{MSY}}$ can be replaced by $\mathrm{MSY}=M 0.5 B_{\mathrm{o}}$.

Considering that the ratio between $B_{o}$ and $B_{\text {MSY }}$ and between $M$ and $F_{M S Y}$ could be different for different species groups, Gulland proposed the following generalized form of eqn. (1):

$\mathrm{MSY}=x M B_{\circ}$

and suggested that $x$ could be estimated from the Beverton and Holt yield tables.

Gulland's equation has been criticized by Francis (1974), Deriso (1982) and Beddington and Cooke (1983), among others, and it is now generally recognized that $F_{\text {MSY }}$ is often lower than $M$ and that eqn. (1) overestimates MSY. Therefore eqn. (2), where $x$ is usually smaller than 0.5 , should be preferred. For instance, Garcia and LeReste (1981) noted that for tropical penaeids the value of $x$ was in the range 0.32-0.44. Beddington and Cooke (1983) give a collection of graphs from which approximate values of $x$ can be read for various combinations of growth parameters, age at first capture and natural mortality. It is worth noting, however, that these two authors and Gulland assume a density-independent recruitment to calculate $x$, in open contradiction of the original Schaefer model.

It should be noted that Gulland's equations are not applicable when significant exploitation is already underway and the virgin biomass cannot be estimated. 


\section{CADIMA'S FORMULA}

A generalized version of Gulland's estimator was proposed by Cadima (in Troadec, 1977) for exploited fish stocks, for which only limited stock assessment data are available. Cadima's estimator has the form

$\mathrm{MSY}=0.5 Z B_{\mathrm{c}}$

where $B_{c}$ is the current average (annual) biomass and $Z$ is the total mortality rate.

Since $Z=F+M$ and $Y_{\mathrm{c}}=F B_{\mathrm{c}}$, the author suggested that in the absence of data on $Z$ the equation could be rewritten as

$\mathrm{MSY}=0.5\left(Y_{\mathrm{c}}+M B_{\mathrm{c}}\right)$

where $Y_{\mathrm{c}}$ is the total current catch and $B_{\mathrm{c}}$ is the estimated current average biomass. As most stocks are now already exploited, this equation is more frequently used in developing (and some developed) fisheries where catch and effort time series are not yet available, but biomass estimates are occasionally obtained from, for instance, trawl or acoustic surveys.

It should be noted that the validity of eqns. (3) and (4) depends on the current level of exploitation. Equation (3) implies that $Z B$ or $0.5(Y+M B)$ remains constant at all levels of $Z$. However, by definition $(Z B)$, the total annual production (i.e. the total biomass dying from natural causes and from fishing) varies with $Z$, as implied by the fundamentals of the production model itself (see Allen, 1971; Csirke and Caddy, 1983). Cadima's estimator is therefore not consistent. Assuming the underlying model (Gulland's formula and the assumption that $F_{\mathrm{MSY}}=M$ ) is correct, Cadima's estimator gives unbiased estimates only when:

(a) the stock is virgin (then $Z=M, B_{\mathrm{c}}=B_{\mathrm{o}}$ and $Y_{\mathrm{c}}=0$, and eqns. (3) and (4) become strictly equivalent to Gulland's estimator for virgin stocks, with the same underlying assumptions);

(b) the stock examined happens to be fished at MSY at the time of the survey for biomass estimates. Then $Z=Z_{\mathrm{MSY}}=F_{\mathrm{MSY}}+M=2 F_{\mathrm{MSY}}, Y_{\mathrm{c}}=\mathrm{MSY}$, and $B_{\mathrm{c}}=B_{\mathrm{MSY}}$, and eqns. (3) and (4), respectively, simplify to $\mathrm{MSY}=0.5 Z_{\mathrm{MSY}} B_{\mathrm{MSY}}=0.5 \times 2 F_{\mathrm{MSY}} B_{\mathrm{MSY}}=F_{\mathrm{MSY}} B_{\mathrm{MSY}}=\mathrm{MSY}$ and

$\mathrm{MSY}=0.5\left(\mathrm{MSY}+M B_{\mathrm{MSY}}\right)=0.5\left(\mathrm{MSY}+F_{\mathrm{MSY}} B_{\mathrm{MSY}}\right)=\mathrm{MSY}$

At any other level of exploitation, Cadima's estimator will be biased if we assume the stock to follow a surplus production model. As illustrated later, the use of Cadima's estimator will either overestimate or underestimate MSY depending on whether the stock is exploited below or above the MSY level.

\section{ALTERNATIVES TO CADIMA'S FORMULA}

Since there is still a need for gross estimators such as the above to be used in data-limited situations, we propose two general equations, derived directly 
from the production models, to estimate the potential yield and the yield curves of exploited fish stocks. These have basically the same foundations and field applications as the Gulland and Cadima estimators, but are more consistent with the underlying models.

The two proposals have been derived based on the two best known and commonly used surplus production models, the Schaefer (1954) model and the Fox (1970) model. Both methods assume that the observations $B_{\mathrm{c}}$ and $Y_{\mathrm{c}}$ are available for one year only. They also assume that $M$ is known and they assume a relationship between $M$ and $F_{\mathrm{MSY}}$ of the form

$M=X F_{\mathrm{MSY}}$

where $X$ is a constant depending on stock parameters.

The two surplus production models may be written as

Schaefer: $Y=a F-b F^{2}$ or $Y / F=a-b F$

Fox: $Y=F \exp (c-d F)$ or $\ln (Y / F)=c-d F$

where $a, b, c$ and $d$ are constants.

As $F=Y / B$ and $Y / F=B$, we can write these surplus production models as follows:

Schaefer: $B=a-b(Y / B)$

Fox: $\ln B=c-d(Y / B)$

If one observation on $B_{\mathrm{c}}$ and $Y_{\mathrm{c}}$ is available and we have a "guesstimate" of $F_{\text {MSY }}^{\prime}($ or $X$ and $M$ ), we can write

Schaefer: $B_{\mathrm{c}}=a-b\left(Y_{\mathrm{c}} / B_{\mathrm{c}}\right)$ and $F_{\mathrm{MSY}}=X M=-a / 2 b$

Fox: $\ln B_{\mathrm{c}}=c-d\left(Y_{\mathrm{c}} / B_{\mathrm{c}}\right)$ and $F_{\mathrm{MSY}}=X M=-1 / d$

These two sets of equations can be solved for $a$ and $b$ in the Schaefer model and $c$ and $d$ in the Fox model to obtain

Schaefer: $a=\frac{2 F_{\mathrm{MSY}} B_{\mathrm{c}}^{2}}{2 F_{\mathrm{MSY}} B_{\mathrm{c}}-Y_{\mathrm{c}}} ; \quad b=\frac{B_{\mathrm{c}}^{2}}{2 F_{\mathrm{MSY}} B_{\mathrm{c}}-Y_{\mathrm{c}}}$

Fox: $c=\ln B_{\mathrm{c}}+Y_{\mathrm{c}} /\left(B_{\mathrm{c}} F_{\mathrm{MSY}}\right) ; \quad d=-1 / F_{\mathrm{MSY}}$

Once we have $(a, b)$ or $(c, d)$ we can draw the yield curves and estimate MSY by

Schaefer: MSY $=-a^{2} / 4 b$

Fox: MSY $=-(1 / d) \exp (c-1)$ 
Thus, one pair of observations $\left(B_{\mathrm{c}}, Y_{\mathrm{c}}\right)$ and assumptions on $M$ and the relationship between $M$ and $F_{\mathrm{MSY}}\left(F_{\mathrm{MSY}}=X M\right)$ are sufficient information to get a first rough estimate of the yield curve and of MSY.

\section{Schaefer model}

Let us assume that the stock in question responds to the Schaefer production model. By replacing $a$ and $b$ in eqn. (6a) by their value in eqn. (5a) we get

$\mathrm{MSY}=\frac{\left(F_{\mathrm{MSY}} B_{\mathrm{c}}\right)^{2}}{2 F_{\mathrm{MSY}} B_{\mathrm{c}}-Y_{\mathrm{c}}}$

In the special case that $F_{\mathrm{MSY}}=M$, the estimator becomes

$\mathrm{MSY}=\frac{\left(M B_{\mathrm{c}}\right)^{2}}{2 M B_{\mathrm{c}}-Y_{\mathrm{c}}}$

If the stock is unfished (i.e. when $F=0, Y_{\mathrm{c}}=0$ and $B_{\mathrm{c}}=B_{\mathrm{o}}$ ), eqn. (7b) becomes Gulland's original equation. It should also be noted that eqn. (7b) and eqn. (4) are equivalent only when $Y_{\mathbf{c}}=M B_{\mathrm{c}}$ and, therefore, when $F=M$. This shows that Cadima's estimator is consistent with Gulland's equation and unbiased at MSY, but gives erroneous values at any other level of exploitation.

It is also worth noting that if $F$ approaches $2 F_{\mathrm{MSY}}$ and $Y$ and $B_{\mathrm{c}}$ approach zero estimators $(7 \mathrm{a})$ and $(7 \mathrm{~b})$ become highly unstable, particularly considering the likely errors in biomass and equilibrium catch. This may cause MSY to vary between plus and minus infinity $( \pm \infty)$, and therefore this estimator is not recommended for high levels of effort or when there is the suspicion that the stock might be strongly overfished (e.g. $F$ approaching $2 F_{\mathrm{MSY}}$ or $2 M$ ).

\section{Fox model}

If the stock in question responds better to the Fox production model, an expression for MSY is obtained by inserting the values of $c$ and $d$ in eqn. (5b) into eqn. (6b)

$\mathrm{MSY}=F_{\mathrm{MSY}} B_{\mathrm{c}} \exp \left(\frac{Y_{\mathrm{c}}}{F_{\mathrm{MSY}} B_{\mathrm{c}}}-1\right)$

In the special case where $F_{\mathrm{MSY}}=M$, the estimator becomes

$\mathrm{MSY}=M B_{\mathrm{c}} \exp \left(\frac{Y_{\mathrm{c}}}{M B_{\mathrm{c}}}-1\right)$

As in the Schaefer model, eqns. (8a) and (8b) have problems of instability when $B_{\mathrm{c}}$ approaches 0 (stock close to extinction), but has the advantage that it happens only at very high (and unrealistic) values of $F$. This estimator can therefore (contrary to eqns. (7a) and (7b)) be used more safely at high levels of fishing.

It should also be noted that eqn. ( $8 \mathrm{~b}$ ) is equivalent to Cadima's formula when $Y_{c}=M B_{c}$ (i.e. MSY), but differs from it at all other levels of $Y_{c}$. 
The performance of the rough estimators for MSY depends on the accuracy of the data used $\left(B_{\mathrm{c}}, Y_{\mathrm{c}}\right)$ and on the appropriateness of the underlying assumptions of the selected estimator. Can the surplus production of the stock be represented as a function of a Schaefer- or a Fox-type model? Would it not be better approached by a simple Beverton and Holt yield per recruit function, i.e. assuming that recruitment is independent of stock size? was the stock really in equilibrium when the biomass was measured? It is, of course, not possible to answer these questions in the absence of long data series, but it is useful to look at the sort of effects one should expect if the assumptions underlying the rough estimator do not correspond to the properties of the stock in question.

To illustrate these questions, three sets of artificial yield and biomass data were simulated under the assumption that the stock behaved as predicted by the Beverton and Holt model, the Schaefer model or the Fox model. Using these simulated observations as input to Cadima's estimator and to the two

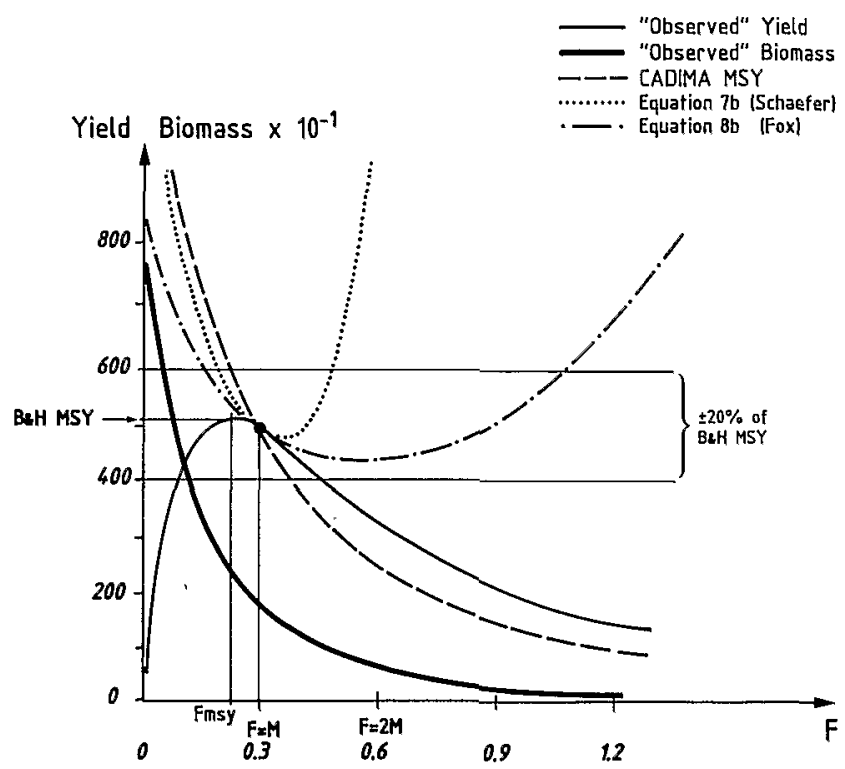

Fig. 1. Comparison between the new MSY estimators based on the Schaefer (eqn. 7b) and Fox (eqn. 8b) models, and Cadima's MSY estimator when the stock follows a Beverton and Holt yield model.

$$
Y=F \exp \left[-M\left(t_{\mathrm{c}}-t_{\mathrm{r}}\right)\right] R W_{\infty}\left[\frac{1}{Z}-\frac{3 S}{Z+K}+\frac{3 S^{2}}{Z+2 K}-\frac{S^{3}}{Z+3 K}\right]
$$

where $S=\exp -K\left(t_{\mathrm{c}}-t_{\mathrm{o}}\right)$. In this model $F_{\mathrm{MSY}}=0.207$ and MSY $=526$ with the following parameters: $K=0.3 ; t_{\mathrm{o}}=0 ; t_{\mathrm{c}}=t_{\mathrm{r}}=0 ; R W_{\infty}=10000$. 
alternative estimators proposed in this paper, we can study their behaviour under various assumptions about the dynamics of the stock.

The simulated yield and biomass curves are shown in Figs. 1-3, which also give the values of the parameters used. To facilitate comparisons, the (arbitrary) parameters behind the simulated data sets are selected so that the yield when $F=M(=0.3)$ takes the same value (the arbitrary value of 500$)$ in all three cases.

When the real stock production function is the Beverton and Holt model (1957), Fig. 1 shows that for $F=M\left(\simeq F_{\mathrm{MSY}}\right.$ ) Cadima's estimator and eqn. (7b) or (8b) give the same results, and these are very close to the "true" MSY as defined by the Beverton and Holt model (Gulland's estimator would give the same result as Cadima's at $F=0$ ). All estimators seriously overestimate the MSY when used at levels of effort below $M$. Beyond $F=M$ the estimators diverge greatly, under or overestimating MSY. If a $20 \%$ error margin is accepted, however, it can be seen that, for instance, the Fox-model-based estimator (eqn. $8 \mathrm{~b}$ ) gives acceptable results within a wider range of $F$ than Cadima's estimator and eqn. (7b). The latter produces extremely high estimates of MSY as $F$ approaches $2 M$ and absurd negative values beyond this point. Similar behaviour of the estimators was observed over a wide range of parameters for the constructed Beverton and Holt model.

When the real production function follows a Schaefer model. Fig. 2 shows that eqn. (7b) finds the correct MSY at all levels of fishing (line A). Cadima's estimator finds MSY for $F=0$ (as does Gulland's estimator) and $F=F_{\mathrm{MSY}}$. At all other levels it gives a biased result. The error is probably acceptable for

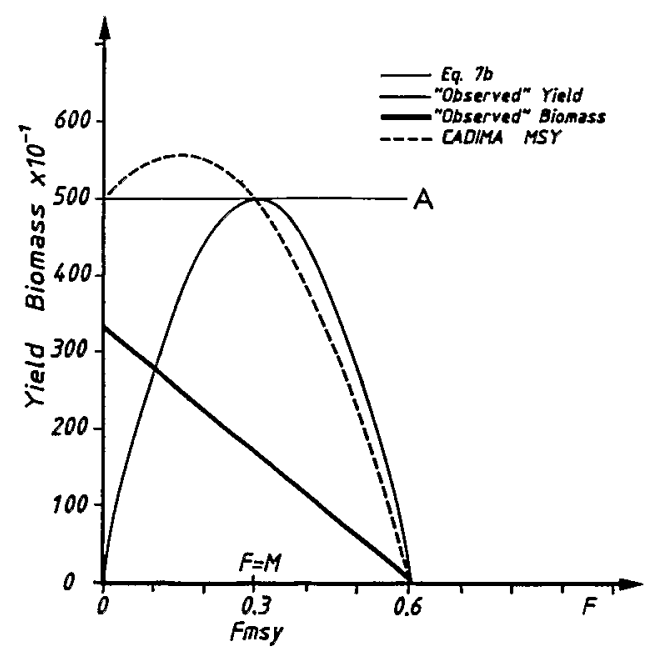

Fig. 2. Comparison between Cadima's MSY estimator and a new MSY estimator (eqn. 7b) where the stocks follow a Schaefer model with the following parameters: $M=0.3 ; a=3333.3 ; b=5555.5$; $\mathrm{MSY}=500$. Note that $a=2 M \mathrm{~b}, b=\mathrm{MSY} / M^{2}$ and eqn. $(7 \mathrm{~b})$ is not defined beyond $F=2 M$. 
$0 \leqslant F \leqslant F_{\mathrm{MSY}}$, considering the other uncertainties in the process, but the performance of Cadima's estimator drops drastically beyond $F_{\text {MSY }}$.

When the real production function is a Fox model, Fig. 3 shows that Cadima's estimator (as well as Gulland's) overestimates MSY when $F=0$. At MSY the former answer is correct. For $F>F_{\text {MSY }}$ MSY is largely underestimated, while eqn. ( $8 \mathrm{~b}$ ) finds the correct MSY at all levels of fishing.

\section{Validation of the estimates}

By definition, a model is a simplified representation of a complex phenomenon used to illustrate one or more processes (in the latter the evolution of total catches as a function of fishing). However, one must bear in mind that even if the model was conceptually correct, using a punctual estimate (such as the stock size $=B_{\mathrm{c}}$ in a given year) to determine the potential yield of a stock and its trajectory under various fishing levels is an adventurous, although sometimes necessary, exercise. Due caution should therefore be taken in selecting an estimator, in validating the estimates and in expressing management or development advice on the basis of such estimators.

When working with the above equations, fishery scientists should check whether the basic assumption of the models are fulfilled. For example, the biomass $\left(B_{\mathbf{c}}\right)$ is meant to be the exploited average biomass, and both the catch and the biomass referred to should have the same age (or size) structure. For instance, the biomass estimate should not include small sizes which are not available to the fishery (as could be the case when using acoustic estimates). This biomass is the annual biomass value and seasonal oscillations caused by

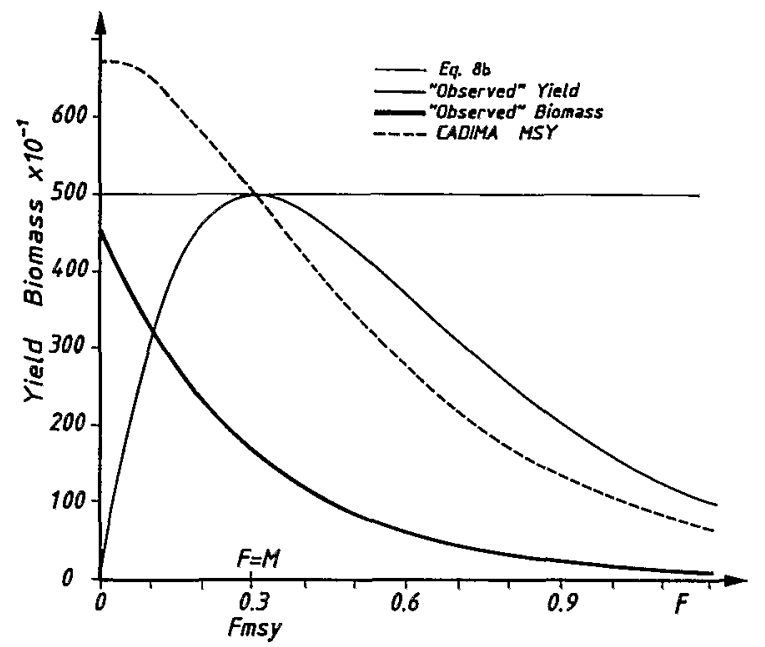

Fig. 3. Comparison between Cadima's MSY estimator and a new MSY estimator (eqn. 8b) where the stocks follow a Fox model with the following parameters: $M=0.3 ; d=(1 / M)=3.3333$; $\mathrm{MSY}=500$. Note that $c=1+\operatorname{Ln}(\mathrm{MSY} / M)$. 
changes in growth, mortality or recruitment, which are likely to be more important in short-living species such as shrimps, squids, anchovies, etc., should be taken into account and as far as possible levelled off to obtain an appropriate annual average of the total biomass. If seasonal oscillations of biomass are caused by migrations, then the peak biomass representing the real stock size should be used. If another country is exploiting the same stock at another season of the year in another area, the total catches should be included in the calculations and the MSY estimate should refer to the whole unit stock.

The estimated potential yield could also be validated by comparison. with other stocks for which information might be available. Some of the questions to be asked are: how does the estimate of MSY $/ \mathrm{km}^{2}$ stand with respect to the estimates for other stocks of the same species in ecologically similar areas, exploited under similar fishing regimes?; does the size structure of the catch provide support for assessments implying that the stock is heavily overfished (e.g. predominance of juveniles) or underfished (e.g. predominance of large, old fish)? It should be noted that a closer look at the length-frequency composition of the catches would give some guidance on relative levels of exploitation, and this should be among the first data to be collected in any developing fishery in order to allow some estimations of total mortality rate for crosschecking with other methods. If the stock in question has been exploited for some time it is likely that a time series of catch data is available, and this should also be examined. Even if no detailed effort data are available, the indication that after a period of sustained increase the total catch has been stable for some time may mean that the MSY has been reached at least for the present regime of exploitation ( $F$ at age), while if the catch has dropped from a previously high level, it may mean that the stock is overfished and an average of the highest catches experienced in the past could provide an independent approximation estimate of MSY. In interpreting catch time series as suggested above, one assumes (and should indeed also check) that such variations in catch are caused by changes in fishing effort and not by causes of environmental or economic origin.

\section{DISCUSSION}

The equations proposed above are straightforward derivations of two wellknown surplus production models (Schaefer, 1954; Fox, 1970, 1974) which have found extended application as well as criticism in the fisheries literature.

Contrary to Cadima's equation, the ones proposed here are consistent with the above models. They are suitable for the specific cases where no long data series of catch or effort are available and where the only estimates available are the total catch, average total biomass and the most likely level of fishing mortality needed to obtain the MSY of the fish stocks in question.

The equations proposed have the same limitations and constraints as the 
models from which they were derived. These have been widely discussed by several authors (see e.g. Larkin, 1977; Sissenwine, 1978; Garcia and LeReste, 1981 ) and the main assumptions are that the biological processes involved are deterministic, the fishery is on a single stock with stable age/size characteristics, the catchability is not density-dependent, and there are no time lags. In addition, the proposed estimators use biomass estimates (as well as $F_{\text {MSY }}$ and $M$ estimates) that are usually poorly estimated or based on educated guesses. This may be particularly critical if no sound auxiliary research data are available to constrain the estimates or assess their reliability.

The comparison of estimators' performance has not exhausted the possible combinations of errors which can be made. The amount and direction of the error depend on the quality of the input data, the level of fishing when the estimate is made, and the degree of coherence between the assumptions underlying the estimator used and the reality of the stock. These examples show that non-consistent estimators, like eqns. (3) and (4), can generate errors, especially beyond $F_{\text {MSY }}$. They also show that estimators which are internally coherent can lead to error if the resource is best described by a different model from the one on which the estimator is based. These differences and their consequences on management decisions could be very important, even for an approximate first estimate. It is therefore advisable to use as many models as possible ( $Y / R$, Fox and Schaefer) in order to have some idea of the degree of uncertainty attached to the MSY estimate and to the shape of the production curve (especially beyond $F_{\text {MSY }}$ ).

Fishery scientists are often asked to express their results in terms usable by managers, and in data-poor conditions this may mean relying, to a great extent, on formulations like the ones proposed here until proper data files are built up and research facilities provided. When providing management advice on the basis of simple estimators, fisheries scientists should stress the frailty of the estimates at hand and the limitations and alternative approaches proposed to the MSY or $F_{\text {MSY }}$ targets as management objectives (see, e.g. Garcia and LeReste, 1981; Caddy and Csirke, 1983), without undermining the usefulness of these estimates as preliminary reference benchmarks.

If the stock is still unexploited or very lightly exploited, a maximum target between $1 / 2$ and $2 / 3$ of the estimated MSY could be set as a preliminary catch figure to aim at in planning development processes. If, however, the stock in question is already being exploited at a relatively high level, then as well as estimating MSY and setting a level of $F_{\text {MSY }}$ (or 2/3 MSY), advice should also be provided on the potential for increase or decrease in fleet size and the consequent likely changes in catch rates. It is unlikely that short-cut methods, such as the ones proposed here, would need to be applied in situations where the stocks are already fished at or beyond MSY, particularly because it is expected that at this point the development of the fishery would have been followed by the development of at least some basic form of fishery research data base, which should allow the application of more elaborate and reliable stock 
assessment methods. It must be pointed out, however, that most of these estimators become highly unreliable at high levels of effort and therefore should not be used under such conditions.

The proposed methods should not be considered as a substitute for the classical catch and effort analysis, but rather as a more robust substitute for the Cadima estimator. It is our opinion that the new proposals, which provide an estimation of MSY as well as a first approximation to the surplus production curve, may offer a better basis for a preliminary management-oriented analysis.

\section{ACKNOWLEDGEMENT}

The authors are grateful to Dr. Ian F. Somers, Division of Fisheries Research, CSIRO, Australia, for his useful and constructive comments on an earlier draft of this paper.

\section{REFERENCES}

Allen, K.R., 1971. Relation between production and biomass. J. Fish. Res. Board Can., 28: 15731581.

Alverson, D.L. and Pereyra, W.T., 1969. Demersal fish exploitations in the Northeastern Pacific Ocean. An evaluation of exploratory fishing methods and analytical approaches to stock sizes and yield forecasts. J. Fish. Res. Board Can., 26: 1985-2001.

Beddington, J. and Cooke, J.G., 1983. The potential yield of fish stocks. FAO Fish. Tech. Pap., 242: $47 \mathrm{pp}$.

Beverton, R.J.H. and Holt, S.J., 1957. On the dynamics of exploited fish populations. Fish. Invest. Minist. Agric. Fish. Food (G.B.) Ser. II Salmon Freshwater Fish.

Caddy, J.F. and Csirke, J., 1983. Approximations to sustainable yield for exploited and unexploited stocks. Oceanogr. Trop., 18(1): 3-15.

Csirke, J. and Caddy, J.F., 1983. Production modeling using mortality estimates. Can. J. Fish. Aquat. Sci., 40: 43-51.

Deriso, R.P., 1982. Relationship of fishing mortality and growth and the level of maximum sustainable yield. Can. J. Fish. Aquat. Sci., 39: 1054-1058.

Fox, W.W.Jr., 1970. An expunential surplus-yield model for optimizing exploited fish populations. Trans. Am. Fish. Soc., 99(1): 80-88.

Fox, W.W., Jr., 1974. An overview of production modelling. Collective Volume of Scientific Papers ICCAT, 3: 142-156.

Francis, R.C., 1974. Relationship of fishing mortality to natural mortality at the level of maximum yield under the logistic stock production model. J. Fish. Res. Board Can., 31: 1539-1542.

Garcia, S. and LeReste, L., 1981. Life cycles, dynamics, exploitation and management of coastal penaeid shrimp stocks. FAO Fish. Tech. Pap., 203: 215 pp.

Gulland, J.A., 1971. The Fish Resources of the Ocean. Fishing News (Books), West Byfleet, 255 pp.

Larkin, P.A., 1977. An epitaph for the concept of Maximum Sustainable Yield. Trans. Am. Fish. Soc., 106(1): 1-11.

Schaefer, M.B., 1954. Some aspects of the dynamics of populations, important for the management of the commercial fisheries. Bull. Inter-American Trop. Tuna Comm., 1(2): 56 pp.

Sissenwine, M.P., 1978. Is MSY an adequate foundation for optimum yield? Fisheries, 3(6): 2224, 37-42.

Tiurin, P.V., 1962. The natural mortality factor and its importance in regulating fisheries. Vopr. Ikhtiol., 2: 403-427.

Troadec, J.-P., 1977. Méthodes semi-quantitatives d'évaluation. FAO Circ. pêches, 701: 131-141. 